\title{
Studying the suppressing effect of mesenchymal stem cells derived from amniotic membrane on colorectal cancer
}

\author{
Niloofar Mesbah ${ }^{1}$, Nooshin Barikrow ${ }^{2, *}$, Masoumeh Heshmati² \\ 1Pharmaceutical Sciences Research Center, Tehran Medical Sciences Branch, Islamic Azad University, Tehran, Iran \\ ${ }^{2}$ Department of Molecular and Cellular Sciences, Faculty of Advanced Sciences and Technology, Tehran Medical Sciences Branch, \\ Islamic Azad University, Tehran, Iran
}

\begin{abstract}
Mesenchymal stem cell therapy is considered as a proper tool for biological activities and treatment of diseases and cancer. Here, the suppressing effect of mesenchymal stem cells derived from the amniotic membrane (AM-MSCs) on colorectal cancer (HT29 cell line) was studied. MSCs were isolated from the human amniotic membrane and identity tests were performed. AM-MSC and HT29 cells were cocultured and MTT assay was used to determine proper inhibitory concentration. The apoptotic effect of AM-MSC on HT29 cells was studied by Acridine orange staining. Expression of CDK2 protein and the antioxidant effect of AM-MSCs on HT29 was performed by immunocyto chemistry test. The effect of MSC on the HT29 cell line migration was determined by Scratch test. The result showed that AM-MSC had proliferation-inhibitory effects and caused apoptosis of the HT29 cell line. Real Time-PCR assay showed the increase of gene expression of p53, Caspase 3 in apoptosis, and p21 in tumor cell line treated with AM-MSC. AM-MSC arrested the cell cycle of tumor cells by reduction of CDK2 protein. The migration of HT29 and ROS expression levels was decreased. Altogether, AM-MSC may play a role in suppressing colorectal cancer HT29 cell line by inhibiting angiogenesis, cell cycle, and induction of apoptosis and has antioxidant properties.
\end{abstract}

Keywords: Amniotic membrane, Colorectal cancer, Co-culture, Mesenchymal stem cell

\section{Introduction}

Colorectal cancer (CRC) is known to be the third deadliest cancer in the world, accounting for 9.7\% of all cancers $[1,2]$. CRC progresses slowly, at first as a mass in the internal rectum, and then continues to grow as unusual growth in the cells, which can then extend to the blood vessels, increasing the chance of distant metastasis to liver and lung sites [1, 3, 4]. Although great progress made in clinical treatment and diagnostic developments, effective treatment for this cancer is now a major challenge because metastasis and recurrence of tumors are two important factors in colorectal cancer [5, 6]. Inhibition of apoptosis and uncontrolled proliferation of tumor cells are considered to be factored in the development of cancer $[5,7,8]$.

There are many ways to treat cancer, including chemotherapy, radiation therapy, and surgery. Due to the side effects of each of these methods, they should be replaced with new therapies [9]. For this reason, cell therapy in various ways over the last few years has been very much considered. In the cell therapy process, a wide array of cells are used, one of the most important is MSCs, mesenchymal stem cell therapy is

\footnotetext{
*Corresponding author:

Nooshin Barikrow, PhD

Department of Molecular and Cellular Sciences,

Faculty of Advanced Sciences and Technology,

Tehran Medical Sciences Branch, Islamic Azad University, Tehran, Iran

Tel/Fax: +98 2188991117

Email: nooshinbarikrow@gmail.com

http://orcid.org/oooo-0001-9367-3885
}

Received: February, 18, 2021

Accepted: November, 08, 2021
(C) The Author(s) 2021
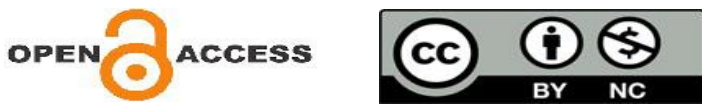
considered as a suitable tool for biological activities, treatment of diseases, and also cancer $[7,10$, 11].

MSCs are multipotent cells, which have selfrenewal ability and differentiate into different cells; they have unique immunological properties that make them a powerful source of clinical treatment $[10,12]$. Among different types of stem cells, mesenchymal stem cells (MSCs) have numerous benefits and can differentiate into different cells or generally to mesodermal and nonmesodermal lineages.

There are many types of non-tumor cells in the tumor microenvironment (TME) that affect the function of the tumor, MSCs are an important part of the TME. They can be used to transmit inhibitory and pro-apoptosis agents to the TME, they communicate with the host cell through the desmosomes, tight junctions, adherens junctions, gap junctions and release antitumor paracrine factors which can cause cancer cell apoptosis and resensitization [12-14]. They play an important role in suppressing cancer tumors by inhibiting angiogenesis, suppressing WNT signaling, and increasing inflammatory infiltration [12].

MSCs can be isolated from a variety of sources such as bone marrow, adipose tissue, cord blood, and amniotic membrane (AM). One of them is the amniotic membrane, access to which is noninvasive, reduced donor damage, less DNA damage, and doesn't decrease in the capacity and the amount of MSCs with the increase in age despite other sources. Also, because of their greater self-renewal ability which differentiates MSCs from other sources, and for a paucity of study on these types of cells, we studied them from this source [15-18]. In recent years, according to the biological roles and specialized capacities of stem cells and amniotic membrane, special attention has been paid to their use in both the medical and clinical settings [11, 19]. AM membrane consists of a thick base membrane layer and an avascular stroma layer; there are two types of stem cells in this membrane, AESCs, and AM-MSC. AMMSCs are located in an avascular stroma. AM membrane is considered to be a postpartum tissue, which can be used for cell therapy due to its antiinflammatory properties, low immunity, antimicrobial, anti-apoptotic, and anti-angiogenesis properties [19-22].
In this research, the effect of MSCs on inhibition of cell cycle was studied by assessing the gene expression of p21, CDK2 protein, and induction of apoptosis by studying the gene expression of p53 and Caspase3, the mechanism of p21protein: it is regulated and activated by $\mathrm{p} 53$, this protein binds and inhibits the activity of CDK2 at the end of the cell cycle in G1 phase, and also Caspase- 3 is activated in the apoptotic cell both by intrinsic and extrinsic pathways. In general, the kinase $(\mathrm{PI} 3 \mathrm{~K}) / \mathrm{AKT}$ and $\mathrm{WNT} / \beta$-catenin signaling pathways control cell survival, proliferation, growth, migration, and cell metabolism. Researches indicated that in some cancers, MSCs exert their apoptotic effects and inhibitory activity by activating the JNK apoptosis signaling pathway and inhibiting the AKT/P13K pathway [23, 24]. Due to the importance of colorectal cancer and the clinical application of AM-MSCs, we study the suppressing effects of AM-MSC on colorectal cancer.

\section{Materials and Methods}

\subsection{Cell line}

The human colon adenocarcinoma HT29 cell line was purchased from the cell bank of Pasteur Institute of Iran. The cells were cultured in a culture medium containing Dulbecco's modified Eagle's medium (DMEM-LG), 10\% bovine serum albumin (BSA), and $5 \%$ penicillin/streptomycin (All from Gibco, USA).

\subsection{MSCs isolation and identification}

The human amniotic membrane was obtained from the cesarean section of the Milad Hospital after obtaining the consent of the pregnant women. All ethical principles were performed in accordance with the declaration of Helsinki and study design approved by Ethical committee of Tehran Medical Sciences Branch, Islamic Azad University. Isolation of mesenchymal stem cells from the amniotic membrane was performed using Alviano's isolation protocol [25]. After isolating, the cells were cultured in DMEM-LG, $\quad 10 \%$ FBS, and 5\% penicillin/streptomycin. Thereafter, cells were trypsinized with $0.25 \%$ Trypsin/EDTA, centrifuged, washed twice with BSA/PBS, and incubated for 30 min with antibodies against CD105, CD90, CD73, CD29 and CD45, CD34. They were conjugated with primary antibodies with PE, FITC, and percP. The negative control staining was used a PE-conjugated mouse IgG1 isotype, FITC-conjugated 
mouse IgG1 isotype antibody and percP-conjugated mouse IgG1 isotype antibody. Flow cytometry was performed with BD FACS Calibur instrument (BD FACSLyric, Germany). The cells were analyzed by flowjo 7.6 [26]. All material is from Sigma Aldrich, USA.

\subsection{Differentiation into osteoblast and adipocyte}

Amniotic mesenchymal cells are adherent cells and are propagated and maintained in tissue culture between 5-10 passages. Cells in passage 6 were prepared for differentiation into osteoblast and adipocytes. In the osteoblast differentiation plate, collagen was first added, after $24 \mathrm{~h}$, collagen was removed. Then AM-MSC cells were added with the differentiation medium in osteoblast and adipocytes plates, after 21 days of differentiation, the alizarin red staining was used to check the differentiation of osteoblast, and the oil red O staining was used to check the adipocytes (All from Sigma Aldrich, USA) [27].

\subsection{MTTassay}

MSC was cultured to study the inhibitory effect of AM-MSC on the proliferation of HT29 cell line. AMMSCs were treated with $50 \mathrm{mg} / \mathrm{ml}$ mitomycin C (MMC; Sigma Aldrich, USA) at $37^{\circ} \mathrm{C}$ for one hour to inhibit the proliferation. The cells were trypsinized and transferred to a 96-well plate. After 24h, HT29 was added to wells with AM-MSC and without AM-MSC. The ratios of AM-MSCs to HT29 were co-cultured as 1:2, 1:5, 1:10 and 1:20; all groups had at least three replicate wells. After 24, 48, 72, and 96 h, $0.5 \mathrm{mg} / \mathrm{mL}$ MTT reagent was added to wells and incubated for $4 \mathrm{~h}$ until a visible purple precipitate was formed, then 150 $\mu \mathrm{l}$ of DMSO was added and shaken for $5 \mathrm{~min}$ at room temperature and absorbance was read at $570 \mathrm{~nm}$ (Sigma Aldrich, USA) [28]. The formula for calculating the inhibitory effect of AM-MSCs on the HT29 cell line is presented below [29].

\section{Formula $=$}

(OD HT29 - OD co-culture) + OD AMMSC/OD HT29 × 100

\subsection{Acridine orange staining $(A O / E B)$}

Acridine orange will stain both live and dead cells and is a vital dye. After preparing co-culture AM-MSC with HT29, the cell culture was removed and washed with PBS, then the cells were fixed with $4 \%$ formaldehyde, $250 \mu \mathrm{L}$ of cell suspension was mixed with $10 \mu \mathrm{L}$ of $\mathrm{AO} / \mathrm{EB}$ solution. The sample should be mixed before quantification and study. Then $10 \mu \mathrm{L}$ mixture was placed on a microscopic slide and examined with the Olympus fluorescence microscope, $400 \mathrm{X}$ objectives [30].

\subsection{Real Time-PCR assay}

Total RNA was extracted from the control HT29 cell, AM-MSC, and co-cultured using the Total RNA Isolation System (Qiagen, USA), and the ratio of absorbance values at 260 and $280 \mathrm{~nm}$ indicated an estimate of RNA purity. In the next step, cDNA was synthesized and samples were compared based on the expression of the GAPDH gene (Qiagen, USA). The primer is determined according to the most appropriate concentration found in the initial tests. Also, deionized distilled water was added until the final volume reached $20 \mu \mathrm{L}$ (Supplementary Table 1). The Real Time-PCR temperature program is shown in Supplementary Table 2. PCR products were subjected to electrophoresis in $2 \%$ agarose gel. Then Real TimePCR was performed to measure the expression level of p21, Caspase3, and p53 genes (Supplementary Table 3).

\subsection{Immunocytochemistry}

As a result of the MTT test, co-culture cells were prepared and fixed with $4 \%$ p-formaldehyde for 20 $\mathrm{min}$ at room temperature and incubated for one hour with blocking buffer which consists of PBS containing $10 \%$ goat serum and $0.3 \%$ Triton $\mathrm{X}-100$. In the next step, the primary antibodies diluted with PBS (1:100, $\mathrm{AB} 32147)$ were added and incubated at $4^{\circ} \mathrm{C}$ for $24 \mathrm{~h}$ in the dark. After washing with PBS, the secondary antibodies diluted (1:150, AB7007) with conjugated FITC were added and incubated for $2 \mathrm{~h}$. Finally, the sample was washed and DAPI was added. The images were captured with a microscope (Olympus BX51, Japan), 400X objective [26].

\section{(ROS) \\ 2.8 Measurement of reactive oxygen species \\ A $1 \mu \mathrm{l}$ of DCFH-DA staining solution was added to1 $\mathrm{ml}$ of the co-culture and HT29 control cells suspension. Cells were mixed and incubated for $15 \mathrm{~min}$ at $37^{\circ} \mathrm{C}$ in the dark, in the next step, cells were washed and resuspended in $300 \mu \mathrm{l}$ of cold PBS, and then ROS generation was monitored using flow cytometry.}




\subsection{Scratch assay}

In this test, the first 6 well-plate of AM-MSC was added, after $24 \mathrm{~h}$ of incubation, HT29 cancer cells were added to AM-MSCs and without AM-MSCs wells. After $72 \mathrm{~h}$, the cells were washed once with PBS buffer, and the DMEM medium containing 10\% FBS was added to the cells. Then with the tip of a pipette, scratches were created on the bottom of the wells. In the next stage, the images were taken by microscope (Olympus BX51, Japan) at the start and after 3 days of the test, the migration of cells from the gap in the coculture and control samples was compared.

\subsection{Statistical analysis}

All experimental data were analyzed with SPSS software version 23 (SPSS Inc., USA). All data are presented as mean \pm standard deviation. For comparison and study of more than two groups, oneway ANOVA was performed, and also values of $\mathrm{P}<$ 0.05 were considered statistically significant.

\section{Results}

3.1 Characterization of cultured AM-MSC

Expressed surface markers of AM-MSC were studied by flow cytometry. The expressed AM-MSC markers are as follows: CD105: 99.8\%; CD90: 81.1\%; CD73: 98.2\%; CD29: 98.5\% and the negative markers are: CD45: 1.22\% and CD34: 2.88\% (Figure 1).
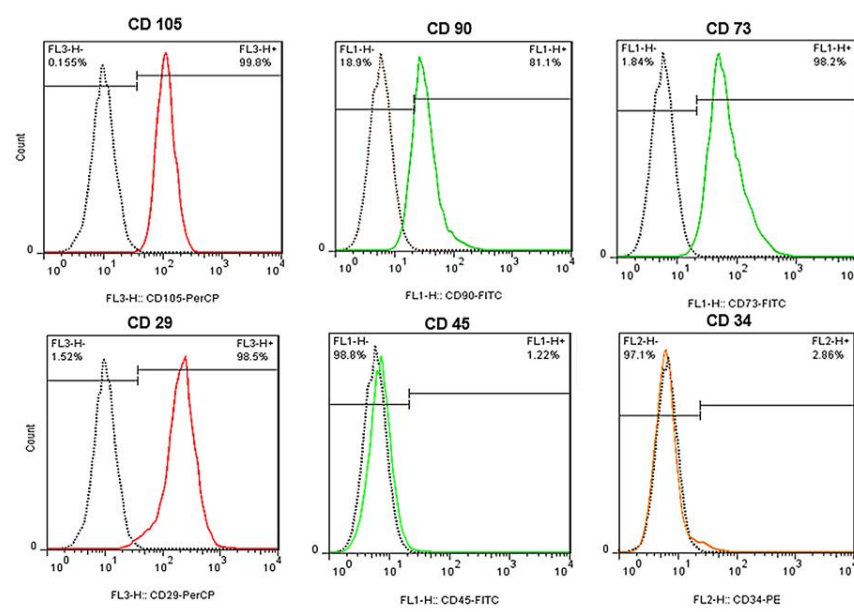

Figure 1. Characterization of cultured AM-MSC, Studied expressed surface marker by flowcytometry.
3.2 Differentiation of AM-MSC into osteoblast and adipocyte

The results showed that AM-MSC cells are affected by differentiation and the culture can differentiate AM-MSC into osteoblast and adipocyte tissue (Figure 2). The cells in passage 5 were exposed to the adipocyte differentiation medium and then stained using Oil Red O, Figures 2 are inverted under a microscope. The red dots are fat vesicles that indicate the differentiation of amniotic membrane stem cells into adipocytes. Also, the cells in passage 5 were exposed to the differentiating medium of the bone and then stained using Alizarin red dye. The Red dots indicate calcium accumulation, indicating differentiation of amniotic membrane stem cells into osteoblast (Figure 2).

\subsection{MTTresult}

MTT assay was used to determine the proper inhibitory concentration in vitro inhibition of HT29 cell growth by AM-MSC. Co-culture AM-MSC with HT29 has performed the ratios 1:2, 1:5, 1:10, and 1:20, at times $24,48,72$, and $96 \mathrm{~h}$, using the MTT method and read Absorbance at $570 \mathrm{~nm}$. Also, the inhibitory effect of MSCs on the viability of tumor cells was expressed as follows: inhibitory rate $(\%)=(\mathrm{OD} 570$ of tumor cells - OD570 of the tumor with MSCs + OD570 of MSCs control)/OD570 of tumor cells x 100\%. The result indicated AM-MSC significantly inhibited of proliferation HT29 cells, a proper concentration of (AM-MSC: HT29) 1:5 for $72 \mathrm{~h}$ was determined (30\%, $\mathrm{p}<0.05$ ). Based on the results AM-MSC showed antiproliferative effects against HT29 cell line (Figure 3).

\subsection{Acridine orange staining}

AM-MSC cells were co-cultured with HT29, acridine orange staining test was performed to determine the apoptotic effect of AM-MSC on HT29 cells. The green area represents living cells and the orange area represents apoptotic cells, the figure indicated apoptosis of cell line HT29 was increased after co-culture with AM-MSC. However, don't occur apoptosis in HT29 and AM-MSC control cells. The results showed that AM-MSC cells induce apoptosis in cell line HT29 (Figure 4). 
Figure 2. Differential AM-MSC to osteoblast and adipocyte. After staining Alizarin red, the pointers indicated calcium accumulation. (A): AM-MSC control cells. (B): AM-MSC differentiated into osteoblast cells. After staining Oil red $\mathrm{O}$, the pointers indicated adipose vesicles. (C): AM-MSC control cells. (D): AM-MSC differentiated into adipose cells ( $X$ 25). The results demonstrated that AM-MSC cells have the ability differentiate to osteoblast and adiposity tissue.
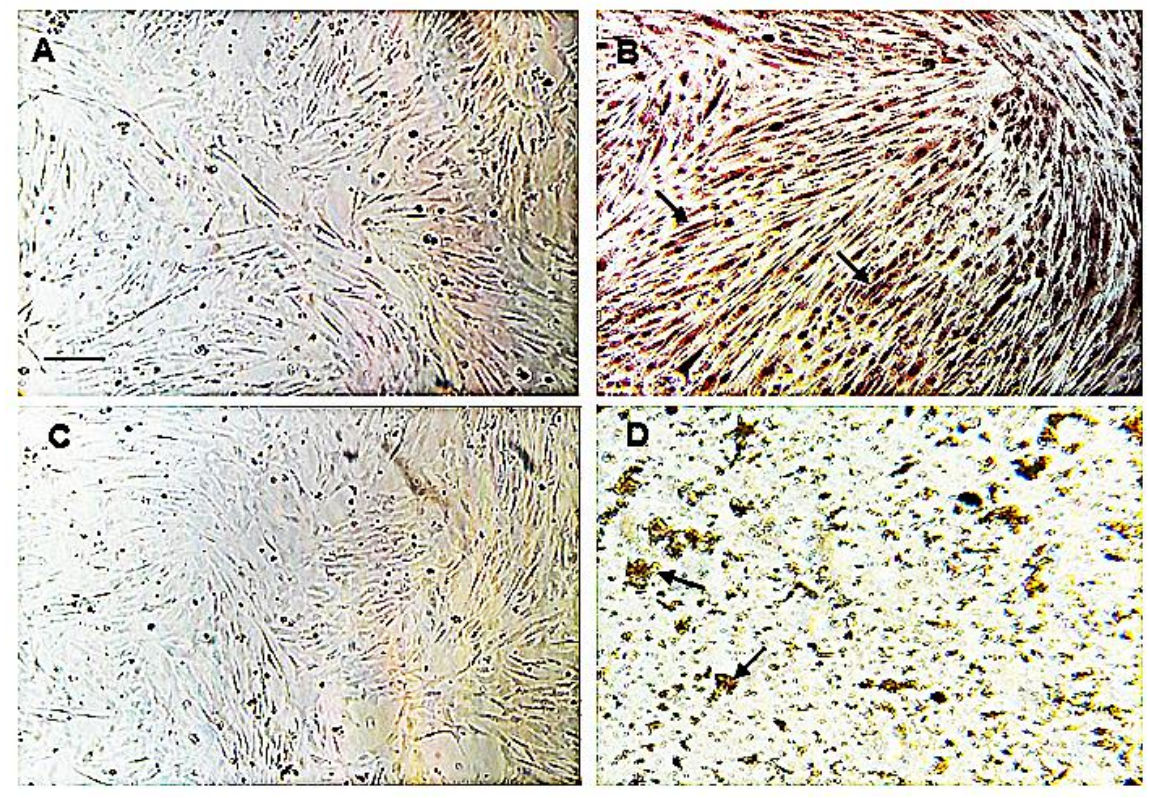

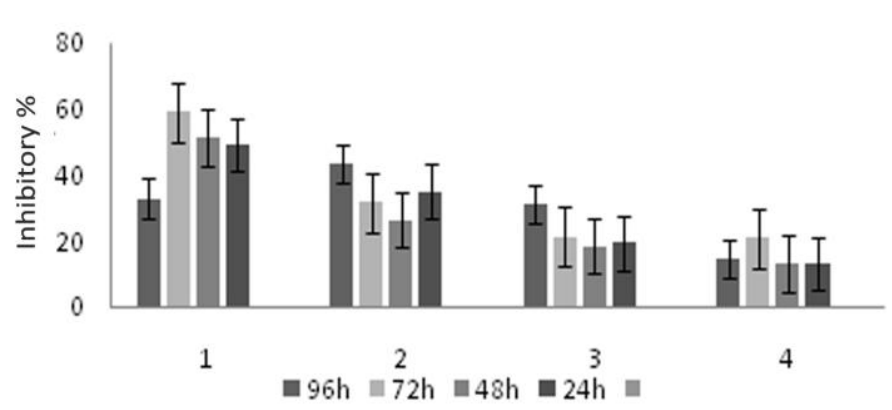

Figure 3. MTT assay was performed to determine the proper inhibitory concentration in vitro inhibition of HT29cell growth by AM-MSC. (AM-MSC: HT29) 1: (1:2), 2: (1:5), 3: (1:10), 4: (1:20). AM-MSC significantly inhibited of HT29 cells, a proper concentration of (AM-MSC: HT29) 1:5 for 72h was determined $(30 \%, \mathrm{P}<0.05)$.

\subsection{Real Time-PCR}

After AM-MSC cells were co-cultured with HT29, to evaluate gene expression of p21, p53, and caspase3 for apoptosis and cell cycle, tumor cells were used. In the Real Time-PCR assay, the GAPDH was control of internal gene expression on all cells group. The results showed that in the co-culture sample, the genes expression of p21: 27.83 fold, p53: 1.64 fold, and also caspase3: 9.25 fold was significantly increased compared with the HT29 control cell line $(\mathrm{P}<0.001)$ (Supplementary Figure 1).

\subsection{Immunocytochemistry}

An immunocytochemistry test was performed to determine the level of CDK2 protein in HT29 cells after co-culture with AM-MSC, the results indicated that the protein expression of CDK2 in tumor cells in the co-culture sample was significantly reduced by 63\% compared with the HT29 control cell line (P $<$ o.001). The results showed that AM-MSC cells inhibited the cell cycle at the end of the G1 phase by reducing $\mathrm{CDK} 2$ on tumor cells (Figure 5).

\subsection{Measuring Reactive oxygen species}

After co-culturing HT29 with AM-MSC, to study the antioxidant properties of AM-MSC on HT29 cell line, the expression of ROS was measured by flow cytometry. The result showed significant reduction of ROS expression in co-cultured cells (Fluorescence amount $=223)$ compared with HT29 control cells (Fluorescence amount $=233)(\mathrm{P}<0.05)($ Figure 6). The results indicated that AM-MSC can induce antioxidant properties by reducing ROS in HT29 tumor cells.

\subsection{Scratch assay}

After co-culturing AM-MSC with HT29, the scratch assay was used to examine the effect of AMMSC on the HT29 cells migration. The scratch test showed that HT29 co-cultured with AM-MSC did not migrate on the wounding space between cells but the HT29 control cell line migrated and filled the wound, 

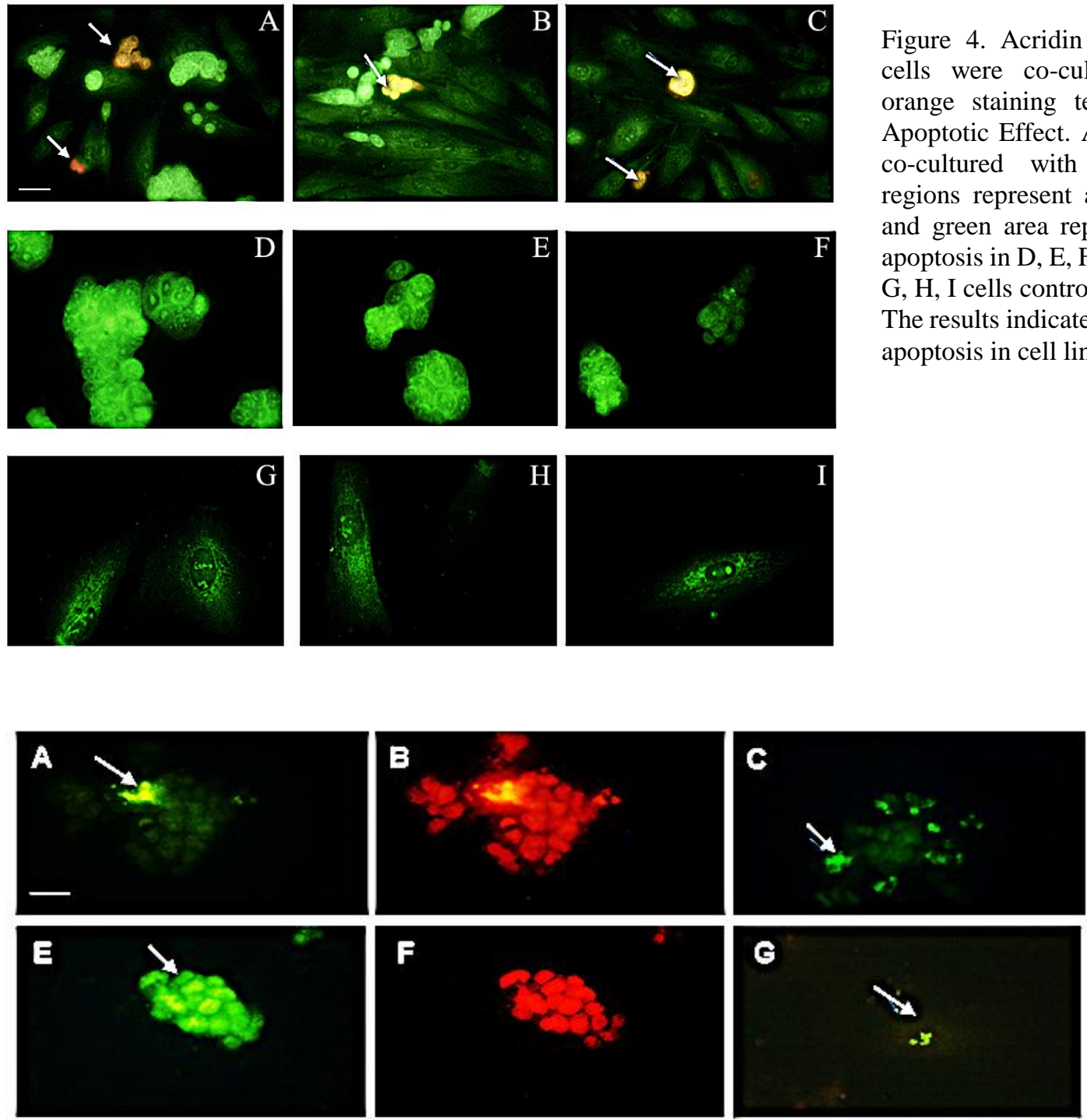

Figure 4. Acridin orange staining. AM-MSC cells were co-cultured with HT29, acridin orange staining test was used to determine Apoptotic Effect. A, B, C: HT29 cell lines are co-cultured with AM-MSC cells. Orange regions represent apoptosis of cell line HT29 and green area represent of living cells, don't apoptosis in D, E, F. The control cells HT29 and $\mathrm{G}, \mathrm{H}$, I cells control the AM-MSC cells (X400). The results indicated that AM-MSC cells induce apoptosis in cell line HT29.

Figure 5. Immunocytochemistry test was used to determine the level expression of CDK2 protein in cell line HT29 after co-culture with AM-MSC. A: co-culture 1 (AM-MSC with HT29) 20\% expression of CDK2, B: PI staining co-culture 1, C: co-culture 2 (AMMSC with HT29) 40\% expression of CDK2, D: PI staining co-culture 2, E: control cell line HT29 90\% expression of CDK2, F: PI staining cell line HT29, G: control AM-MSC with $40 \%$ expression of CDK2, H: PI staining AM-MSC (X400). The results showed AM-MSC cells inhibition cell cycle by reduced CDK2 on tumor cells HT29.

the result indicated that AM-MSCs inhibited HT29 cell migration (Figure 7).

\section{Discussion}

Colorectal cancer is known as one of the deadliest cancer, despite the great medical progress, there is no effective treatment for this cancer. In this research, we examined the suppressing effect of AM-MSC on HT29 cell line, studied the effective induction of apoptosis, cell cycle arrest, migration, and antioxidant properties of AM-MSC cells on HT29 cells. The results showed that AM-MSC cells induced apoptosis by increasing the expression of the Caspase 3 and p53, inhibiting the cell cycle by increasing the expression of the p21 gene and decreasing the $\mathrm{CDK} 2$ protein. Furthermore, these results showed that AM-MSC cells inhibited the migration of cancer cells and the induction of antioxidant activity to HT29 cancer cells by reducing the amount of ROS.

MSCs are multipotent cells, which have selfrenewal ability and can differentiate into other tissues. AM-MSCs have unique immunological properties and 

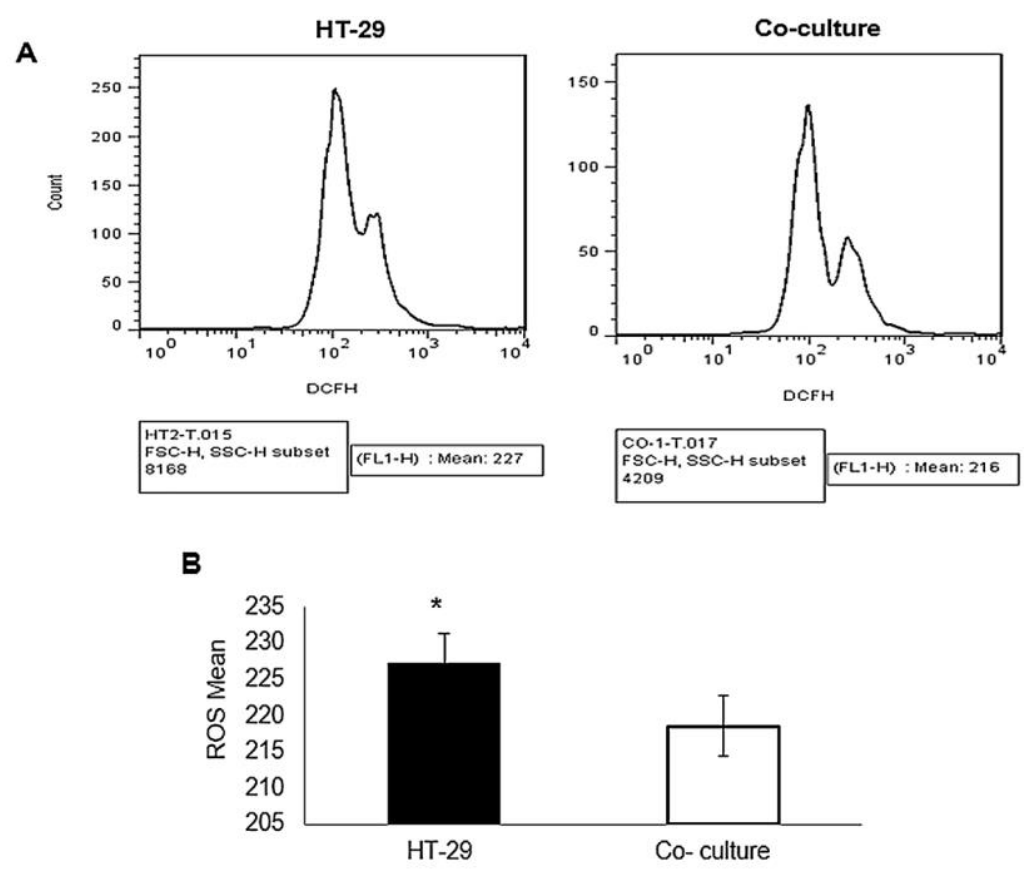

Figure 6. Measuring ROS. After co-cultured HT29 with AM-MSC, to examined the antioxidant properties of AM-MSC Measuring expression ROS by flowcytometry. A: flowcytometry in HT29 control cells, and coculture cells. B: Represents a reduction in the amount of ROS in co-culture cells compared with HT29 control cells. ROS mean of 233 indicate the fluorescence amount in control cells HT29, and 223 fluorescence amount in coculture cells. The results indicate AM-MSC induce antioxidant properties by reduce ROS in tumor cells HT29. $*=\mathrm{P}<0.05$
Figure 7. Scratch assay. After co-cultured AMMSC with HT29 the Scratch test was used to evaluate the effect of AM-MSC on the cell line HT29 migration. A: AM-MSC; B: cell line HT29. A: co-culture 0 day, B: co-culture after 3 day, C: cell line HT29 0 day, D: cell line HT29 after 3 day (X10). The result indicated cell line HT29 treatment with AM-AM-MSC wasn't migrate on the wounding space between cells but control cell line migrated and filled wound, AM-MSCs inhibited the HT29 cell migration.
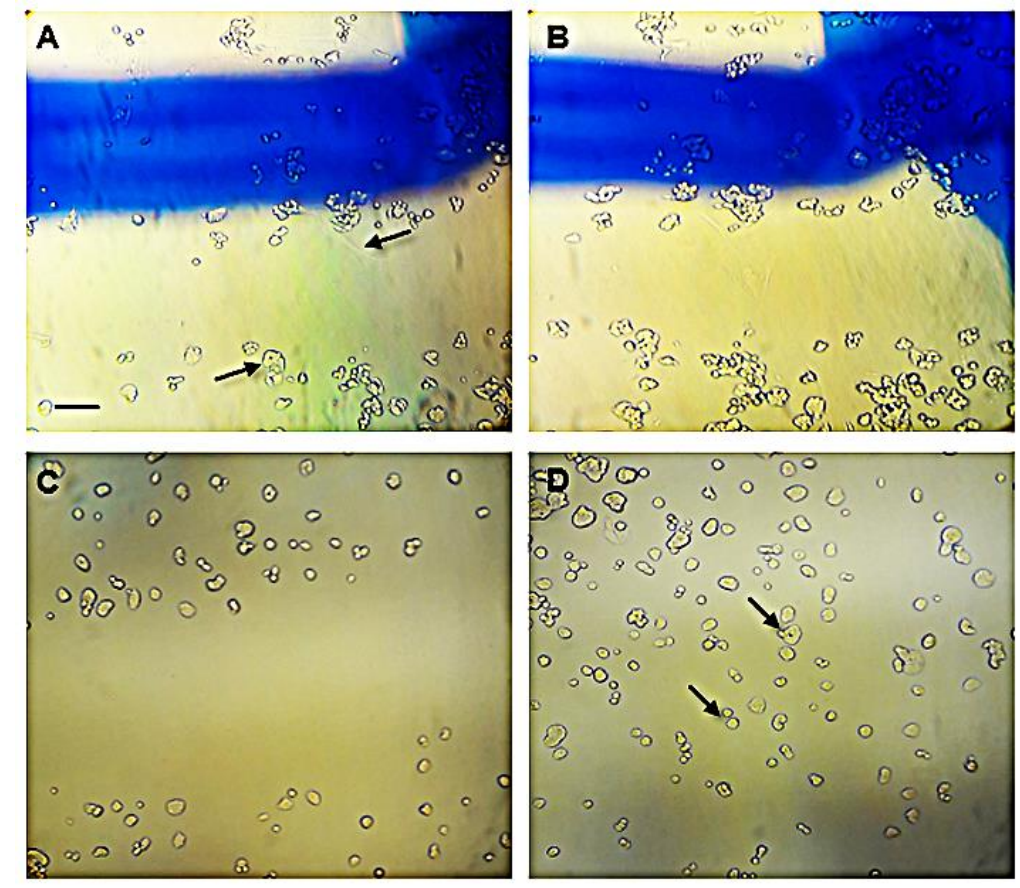

don't reduce in the capacity and the amount of MSCs with the increase in age despite other sources and also because of the greater self-renewal ability and differentiation of MSCs from this source compared to others, this cell is suitable for the study of cell therapy $[10,15,16]$.

However, the role of MSC hasn't been determined about the support or suppressing of the tumor cell, but it may be attributed to tumor models, the animal host, the dose or timing of the MSCs injected, and might be due to their activation status [31, 32]. According to previous studies, MSC causes resensitization and cancer cell apoptosis by releasing antitumor paracrine factors that seek to release the chemoattractants from the tumor. Also, MSCs with the secretion of interleukin and interferons inhibit 
cancer cells, IL-18, which inhibits proliferation and cell division in tumor cells, IL-15, which also inhibits the growth of tumor cell cells also leads to NK Cell, CD8+, and lymphocyte $\mathrm{T}$, and causes the immune system to react in tumor cells $[14,33,34]$. In addition, MSC helps in the inhibition of tumors by inhibiting tumor proteins, activating various signaling pathways, and inducing apoptosis in tumor cells. MSCs, by activating the JNK pathway and inhibiting the AKT/PK13 pathway, induced apoptosis in cancer cells [35, 36].

Abd-Allah et al. found that MSC can arrest cell cycle HCC cell line. The expression levels of p21 and p53 were down-regulated in the HCC cell line treatment with MSC [36].

$\mathrm{Lu}$ et al. examined the effect of mesenchymal cells isolated from bone marrow on the cell cycle of mouse H22 cancer cells. P21 gene expression was increased. This is similar to our result in this study. The results of fold increase in p21 gene expression in HT29 cancer cells compared to controls [29].

Also, Ahn et al. studied the effect of MSCs derived adipose tissue on melanoma cancer cells; they found that AT-MSC-CM inhibited melanoma cells by arresting cell-cycle and inducing apoptosis [37]. Hou et al. examined the suppressing effect of BM-MSC cells on HepG2 Hepatic cancer cells, they found that BMMSC inhibits the proliferation and promotes the apoptosis of HepG2 cells by down-regulating the gene expression of bcl-2, cMyc, $\beta$-catenin, and survivin. Also, BM-MSC inhibits the Wnt signaling in tumor cells by secreting Dkk-1 [28]. BM-MSC-CM promotes colorectal cancer (cell line HCT116) progression through AMPK/mTOR-mediated NF- $\mathrm{kB}$ activation and induces proliferation and survival by downregulating p21, p16, p53, BAX and increasing protein expression of Bcl2 [38]. Further, these results of AM-MSC cells inhibited the migration of cancer cells and the induction of antioxidant activity to HT29 cancer cells by reducing the amount of ROS.

The role of MSCs has not been determined regarding the support or suppressing of the tumor cell but because the MSCs are an important part of the TME, they release antitumor factors, and also in this research, the dose and timing of the MSCs were determined. The result indicated that AM-MSC can play a role in suppressing colorectal cancer (HT29 cell line) by inhibiting angiogenesis, suppressing the cell cycle, induction of apoptosis, and antioxidant properties. Also, these cells, have an antitumor effect in acceptable medical ethical issue and non-invasive access are an appropriate option for the treatment of some cancers and proper cell for cell therapy, so further studies in this field seem to be necessary.

\section{Supplementary files}

Supplementary file 1 .

\section{Acknowledgements}

This research was supported by Pharmaceutical Sciences Research Center, Tehran Medical Sciences Branch, Islamic Azad University, and Tehran, Iran. We wish to thank Nasim Iran Doost and Parisa Nikkhoo for their supports.

\section{Author contributions}

The authors were equally involved in data collection and writing the article. Also, all authors approved the final version of the manuscript.

\section{Conflict of interests}

There is no conflict of interest.

\section{Ethical declarations}

All ethical principles were performed in accordance with the declaration of Helsinki and study design approved by Ethical committee of Tehran Medical Sciences Branch, Islamic Azad University (IR.IAU.PS.REC.1396.131). Also, a signed informed consent was obtained from the patients fortaking blood samples.

\section{Financial Support}

This research was supported by Faculty of Advanced Science and Technology, Tehran Medical Sciences Branch, Islamic Azad University, and Tehran, Iran (Registered number: 1087).

\section{References}

1. Marley AR, Nan H. Epidemiology of colorectal cancer. Int J Mol Epidemiol Genet. 2016; 7(3):105-14.

2. Pan T, Xu J, Zhu Y. Self-renewal molecular mechanisms of colorectal cancer stem cells. Int J Mol Med. 2017; 39(1):9-20.

3. Valastyan S, Weinberg RA. Tumor metastasis: molecular insights and evolving paradigms. Cell. 2011; 147(2):275-92.

4. Assi R, Mukherji D, Haydar A, Saroufim M, Temraz S, ShamseddineA. Metastatic colorectal cancer presenting with bone marrow metastasis: a case series and review of literature. $\mathrm{J}$ Gastrointest Oncol. 2016; 7(2):284-97. 
5. Garza-Treviño EN, Said-Fernández SL, Martínez-Rodríguez HG. Understanding the colon cancer stem cells and perspectives on treatment. Cancer Cell Int. 2015; 15(1):2.

6. Huang Q, Li S, Cheng P, Deng M, He X, Wang Z, et al. High expression of anti-apoptotic protein Bcl-2 is a good prognostic factor in colorectal cancer: Result of a meta-analysis. World $\mathrm{J}$ Gastroenterol. 2017; 23(27):5018-33.

7. Lopez J, Tait SW. Mitochondrial apoptosis: killing cancer using the enemy within. Br J Cancer. 2015; 112(6):957-62.

8. Otto T, Sicinski P. Cell cycle proteins as promising targets in cancer therapy. Nat Rev Cancer. 2017; 17(2):93-115.

9. Sage EK, Thakrar RM, Janes SM. Genetically modified mesenchymal stromal cells in cancer therapy. Cytotherapy. 2016; 18(11):1435-45.

10. Shin TH, Kim HS, Choi SW, Kang KS. Mesenchymal Stem Cell Therapy for Inflammatory Skin Diseases: Clinical Potential and Mode of Action. Int J Mol Sci. 2017; 18(2):244.

11. Mohan R, Bajaj A, Gundappa M. Human Amnion Membrane: Potential Applications in Oral and Periodontal Field. J Int Soc Prev Community Dent. 2017; 7(1):15-21.

12. Rhee KJ, Lee JI, Eom YW. Mesenchymal Stem Cell-Mediated Effects of Tumor Support or Suppression. Int J Mol Sci. 2015; 16(12):30015-33.

13. Zhang C, Yang SJ, Wen Q, Zhong JF, Chen XL, Stucky A, et al. Human-derived normal mesenchymal stem/stromal cells in anticancer therapies. J Cancer. 2017; 8(1):85-96.

14. Rivera-Cruz CM, Shearer JJ, Figueiredo Neto M, Figueiredo ML. The Immunomodulatory Effects of Mesenchymal Stem Cell Polarization within the Tumor Microenvironment Niche. Stem Cells Int. 2017;2017:4015039.

15. Nagamura-Inoue $T$, He $H$. Umbilical cord-derived mesenchymal stem cells: Their advantages and potential clinical utility. World J Stem Cells. 2014; 6(2):195-202.

16. Larijani B, Aghayan HR, Goodarzi P, Arjmand B. GMP-grade human fetal liver-derived mesenchymal stem cells for clinical transplantation. Methods Mol Biol. 2015; 1283:123-36.

17. Kim EY, Lee KB, Kim MK. The potential of mesenchymal stem cells derived from amniotic membrane and amniotic fluid for neuronal regenerative therapy. BMB Rep. 2014; 47(3):135-40.

18. Kazemnejad S, Khanmohammadi M, Zarnani A-H, Bolouri MR. Characteristics of Mesenchymal Stem Cells Derived from Amniotic Membrane: A Potential Candidate for Stem Cell-Based Therapy. Perinatal Tissue-Derived Stem Cells: Springer; 2016. p. 137-69.

19. Khayat-Khoei M. Will the Amniotic Membrane and Its Stem Cells Preserve their Capacity after Cryopreservation. J Cytol Histol. 2016; 7:e118.

20. Khayat-Khoei M. Role of Stem Cells in the Anti-tumor Effects of the Human Amniotic Membrane. J Cytol Histol. 2015; 6(6):1.

21. Larson A, Gallichio V. Amniotic derived stem cells: role and function in regenerative medicine. J Cell Sci Ther. 2017; 8(3):1-10. 22. Pozzobon M, Piccoli M, De Coppi P. Stem cells from fetal membranes and amniotic fluid: markers for cell isolation and therapy. Cell Tissue Bank. 2014; 15(2):199-211.

23. Kim EM, Jung CH, Kim J, Hwang SG, Park JK, Um HD. The p53/p21 Complex Regulates Cancer Cell Invasion and Apoptosis by Targeting Bcl-2 Family Proteins. Cancer Res. 2017; 77(11):3092100.

24. Czabotar PE, Lessene G, Strasser A, Adams JM. Control of apoptosis by the BCL-2 protein family: implications for physiology and therapy. Nat Rev Mol Cell Biol. 2014; 15(1):49-63.

25. Díaz-Prado S, Muiños-López E, Hermida-Gómez T, RendalVázquez ME, Fuentes-Boquete I, de Toro FJ, et al. Isolation and characterization of mesenchymal stem cells from human amniotic membrane. Tissue Eng Part C Methods. 2011; 17(1):49-59.

26. Mostafavi FS, Razavi S, Mardani M, Esfandiari E, Esfahani HZ, Kazemi M. Comparative Study of Microtubule-associated Protein2 and Glial Fibrillary Acidic Proteins during Neural Induction of Human Bone Marrow Mesenchymal Stem Cells and AdiposeDerived Stem Cells. Int J Prev Med. 2014; 5(5):584-95.

27. Hanna H, Mir LM, Andre FM. In vitro osteoblastic differentiation of mesenchymal stem cells generates cell layers with distinct properties. 2018; 9(1):203.

28. Hou L, Wang X, Zhou Y, Ma H, Wang Z, He J, et al. Inhibitory effect and mechanism of mesenchymal stem cells on liver cancer cells. Tumour Biol. 2014; 35(2):1239-50.

29. Lu YR, Yuan Y, Wang XJ, Wei LL, Chen YN, Cong C, et al. The growth inhibitory effect of mesenchymal stem cells on tumor cells in vitro and in vivo. Cancer Biol Ther. 2008; 7(2):245-51.

30. Kasibhatla S, Amarante-Mendes GP, Finucane D, Brunner T, Bossy-Wetzel E, Green DR. Acridine Orange/Ethidium Bromide (AO/EB) Staining to Detect Apoptosis. CSH Protoc. 2006(3):pdb.prot4493.

31. Klopp AH, Gupta A, Spaeth E, Andreeff M, Marini F, 3rd. Concise review: Dissecting a discrepancy in the literature: do mesenchymal stem cells support or suppress tumor growth? Stem Cells. 2011; 29(1):11-9.

32. Chulpanova DS, Kitaeva KV, Tazetdinova LG, James V, Rizvanov AA, Solovyeva VV. Application of Mesenchymal Stem Cells for Therapeutic Agent Delivery in Anti-tumor Treatment. Front Pharmacol. 2018; 9:259.

33. Liu X, Hu J, Sun S, Li F, Cao W, Wang YU, et al. Mesenchymal stem cells expressing interleukin-18 suppress breast cancer cells in vitro. Exp Ther Med. 2015; 9(4):1192-200.

34. Trivanović D, Krstić J, Djordjević IO, Mojsilović S. The Roles of Mesenchymal Stromal/Stem Cells in Tumor Microenvironment Associated with Inflammation. Mediators Inflamm. 2016;2016:7314016.

35. Sun Z, Wang S, Zhao RC. The roles of mesenchymal stem cells in tumor inflammatory microenvironment. J Hematol Oncol. 2014; 7:14.

36. Abd-Allah SH, Shalaby SM, El-Shal AS, Elkader EA, Hussein S, Emam E, et al. Effect of bone marrow-derived mesenchymal stromal cells on hepatoma. Cytotherapy. 2014; 16(9):1197-206. 37. Ahn JO, Coh YR, Lee HW, Shin IS, Kang SK, Youn HY. Human adipose tissue-derived mesenchymal stem cells inhibit melanoma growth in vitro and in vivo. Anticancer Res. 2015; 35(1):159-68.

38. Ramasamy R, Lam EW, Soeiro I, Tisato V, Bonnet D, Dazzi F. Mesenchymal stem cells inhibit proliferation and apoptosis of tumor cells: impact on in vivo tumor growth. Leukemia. 2007; 21(2):304-10. 